\title{
NUCLEOSYNTHESIS IN RED GIANT STARS
}

\author{
NAMI MOWLAVI \\ Geneva Observatory \\ CH-1290 Sauverny, Switzerland
}

\begin{abstract}
The production of elements from helium-3 to fluorine in lowand intermediate-mass stars is reviewed and compared to chemical abundances observed at the surface of both red giant branch and asymptotic giant branch stars. It is highlighted that, while the trends predicted by standard models are generally well confirmed, many chemical abundances observed at the surface of red giants require the operation of non-standard mixing in the stellar interior. In addition, chemical abundance predictions from presently available asymptotic giant branch models further suffer from the uncertainties affecting the third dredge-up phenomenon, the source of neutrons and the hot bottom burning process.
\end{abstract}

\section{Introduction}

The progenitors of the red giant stars considered in this paper are those with masses $M$ less than 6 to $8 \mathrm{M}_{\odot}{ }^{1}$ which end their life as white dwarfs (WDs). Considering that their age must be lower than that of the Galaxy, their initial mass is moreover restricted to $\mathrm{M} \gtrsim 0.8 \mathrm{M}_{\odot}$.

The different phases of the evolution of these low- and intermediate-mass (LIM) stars are summarized in [57], to which I refer for a general introduction to the present paper. Let me recall that the surface abundances of LIM stars are altered by several dredge-up episodes during which the convective envelope penetrates into the deep regions and mixes to the surface matter processed by nuclear burning. The so-called 'first' and 'second' dredge-ups bring to the surface ashes from hydrogen-burning nucleosynthesis. The first dredge-up (1DUP) occurs after the main sequence phase when the star enters the red giant branch (RGB) phase, and the second dredge-up after the

\footnotetext{
${ }^{1}$ The case of stars more massive than 6 to $8 \mathrm{M}_{\odot}$ is reviewed by D. Arnett and $\mathrm{K}$. Nomoto in this volume.
} 
core He-burning phase (but only for stars more massive than $\sim 4 \mathrm{M}_{\odot}$ ) when the star enters the asymptotic giant branch (AGB) phase. In contrast, the so-called 'third' dredge-ups (3DUPs) have the distinctive characteristics of bringing to the surface, in addition to the ashes from the H-burning shell, products from helium burning. These 3DUPs occur after thermal instabilities (called 'pulses') which develop recurrently in the He-burning shell of AGB stars. Finally, the abundances at the surface of AGB stars can further be altered directly by nucleosynthesis operating in the envelope when the temperature at its base increases above $50 \times 10^{6} \mathrm{~K}$. This scenario is called hot bottom burning (HBB).

In the context of cosmic chemical evolution, LIM stars are important actors with respect to elements from $\mathrm{He}$ to $\mathrm{Al}$ and to the main component of the s-process elements (which are synthesized by neutron capture on iron and heavier elements, see [3]). This derives from three factors: (a) the rich nucleosynthesis processes occurring in these stars, and especially during the AGB phase ([57]); (b) the high mass loss rates characterizing their late phases of evolution, through which a large fraction of the stellar material is released into the interstellar medium (from about $40 \%$ for a $1 \mathrm{M}_{\odot}$ star to more than $80 \%$ for LIM stars with $\left.\mathrm{M} \gtrsim 4 \mathrm{M}_{\odot}\right) ;(c)$ the initial mass function which favors the formation of LIM stars over massive ones in stellar populations.

Ample observational evidences of the nucleosynthesis operating in LIM stars are available in the literature (e.g. [78]). In this paper, I review the status of nuclides from ${ }^{3} \mathrm{He}$ to ${ }^{19} \mathrm{~F}$ regarding their nucleosynthesis in LIM stars. The cases of ${ }^{7} \mathrm{Li}$ and ${ }^{23} \mathrm{Na}$ are discussed in [59]. The surface abundance predictions from 'standard' stellar calculations are briefly presented in Sect. 2. Section 3 then focuses on the light element ${ }^{3} \mathrm{He}$, Sect. 4 on the CNO elements, and Sect. 5 on fluorine. For each of these nuclides, their nucleosynthesis path is recalled and their predicted abundances in red giants and AGB stars compared to observations. Conclusions and final remarks are drawn in Sect. 6.

\section{Standard model predictions}

'Standard' stellar model calculations refer to those using the Schwarzschild criterion to define the limits of convection boundaries, with no other mixing process taken into account, such as overshooting, diffusion or meridional circulation. The surface abundances predicted by such calculations after the 1DUP are widely documented in the literature, at least for the main nuclides (e.g. [23, 76, 86, 17, 31]). They are summarized in Fig. 1 for Pop. I stars from 0.8 to $10 \mathrm{M}_{\odot}$.

Chemical yields from AGB models are more delicate to predict. Full 
SURFACE ABUNDANCES AFTER $1^{\text {st }}$ DREDGE-UP

'standard' predictions $\mathrm{X}=0.707 \mathrm{Y}=0.275 \mathrm{Z}=0.018$
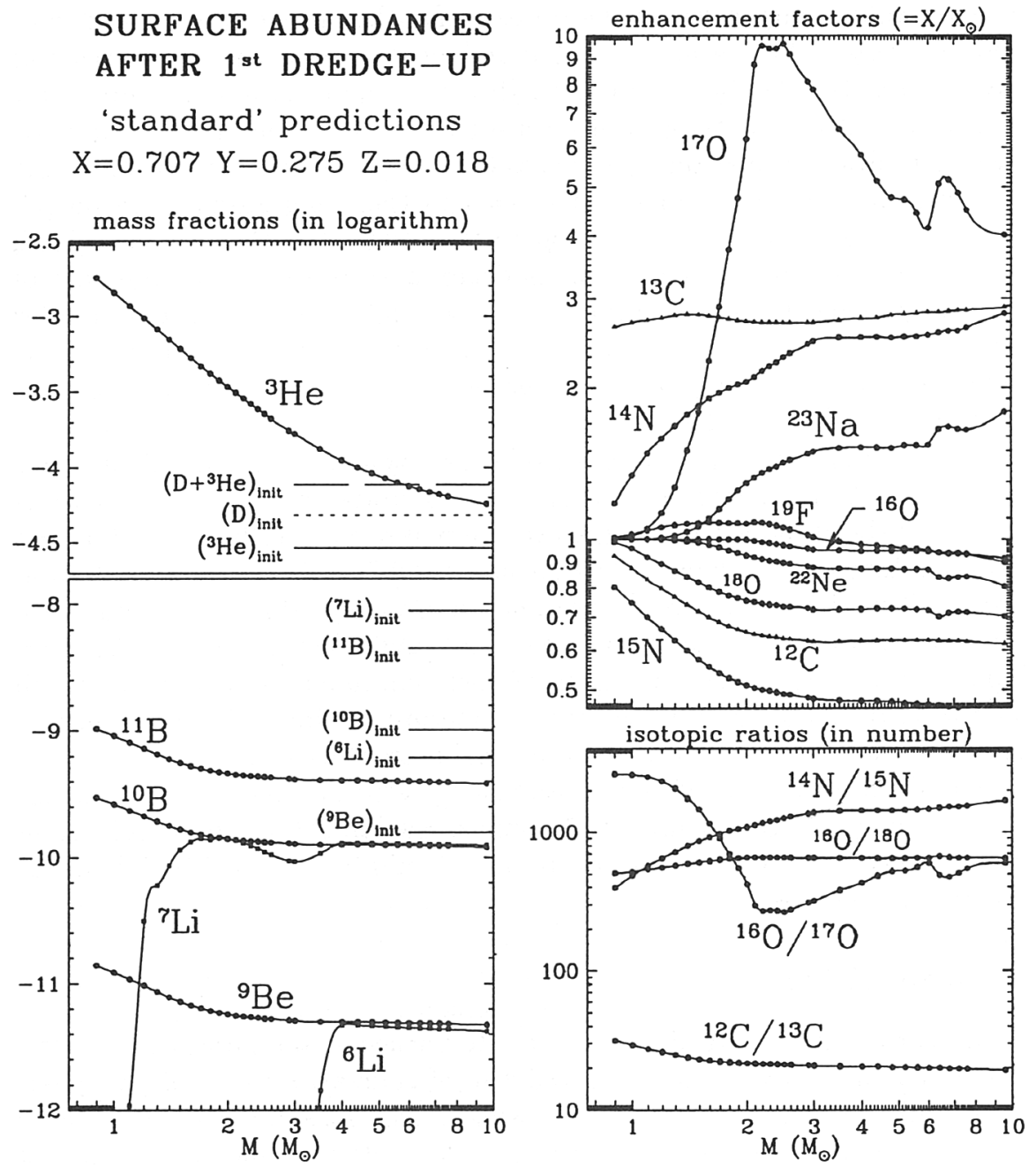

Figure 1. Abundance of elements from ${ }^{3} \mathrm{He}$ to ${ }^{23} \mathrm{Na}$ predicted at the surface of Pop. I red giant stars by standard (i.e. without any extra-mixing beyond the Schwarzschild boundaries for convection) models. The abundances of the $\mathrm{Mg}$ and $\mathrm{Al}$ isotopes, and of other nuclides not reported on the figures, remain essentially solar during the first dredge-up of Pop. I model models. The stellar evolution code is described in Mowlavi (1995,[56]), modified, among others, to use the OPAL equation of state [73], and the Canuto-Goldman-Mazzitelli prescription for convection [22] (with $K_{0}=1.7$ and $\left.\alpha^{*}=0.09\right)$. All models are evolved from the pre-main sequence 'Birth Line' (see e.g. [11]), obtained by accreting $10^{-5} \mathrm{M}_{\odot \mathrm{y}^{-1}}$ on an initial $0.7 \mathrm{M}_{\odot}$ model. The p-capture reaction rates are identical to those used in [60], except for ${ }^{17} \mathrm{O}(\mathrm{p}, \alpha){ }^{14} \mathrm{~N}$ and ${ }^{17} \mathrm{O}(\mathrm{p}, \gamma){ }^{14} \mathrm{~N}$ which are from $[12],{ }^{20} \mathrm{Ne}(\mathrm{p}, \gamma){ }^{21} \mathrm{Na},{ }^{21} \mathrm{Ne}(\mathrm{p}, \gamma){ }^{22} \mathrm{Na},{ }^{22} \mathrm{Ne}(\mathrm{p}, \gamma){ }^{23} \mathrm{Na}$, ${ }^{23} \mathrm{Na}(\mathrm{p}, \gamma){ }^{24} \mathrm{Mg}$ and ${ }^{23} \mathrm{Na}(\mathrm{p}, \alpha){ }^{20} \mathrm{Ne}$ from [32], and ${ }^{22} \mathrm{Na}(\mathrm{p}, \gamma){ }^{23} \mathrm{Mg},{ }^{25} \mathrm{Mg}(\mathrm{p}, \gamma){ }^{26} \mathrm{Al}$ and ${ }^{26} \mathrm{Al}^{\mathrm{g}}(\mathrm{p}, \gamma){ }^{27} \mathrm{Si}$ from [26]. The reader is referred to [4] for a discussion on the uncertainties affecting the $\mathrm{H}$-burning reaction rates. 
stellar evolutionary calculations do exist for that phase, and indeed provide precious information on the abundances expected at the surface of AGB stars $([14,88,16,13,85,35])$. However, these standard models have many difficulties to predict self-consistently surface abundances compatible with observational data. For example, AGB models fail to reproduce the observed properties of $\mathrm{C}$ stars, such as their luminosity function (cf. the 'C star mystery', [49]). The operation of the 3DUP mechanism itself was a puzzling problem to stellar modelers until recently. The presence of s-process elements at the surface of S, SC and C stars presents another difficulty (cf. the 'S star mystery', [74]). Fortunately, the situation looks more promising today ([47]). The interested reader is referred to [58] for further discussion on these issues.

Because of these difficulties encountered by current AGB calculations, 'synthetic' AGB models have been used to study the properties of AGB stars. Synthetic models follow the evolution of several stellar characteristics (surface luminosity, chemical composition, ...) based on relations derived from full model calculations (core mass-luminosity relation, ...), but with the use of several parameters (mass loss rate, efficiency of the 3DUPS, mixing parameters, ...). Examples of such synthetic models are found in $[68,55,87]$. These are very useful to determine values of the parameters necessary to reproduce given observational properties of AGB stars (such as the luminosity function of carbon stars, [87]), which in turn point out on the shortcomings of present full AGB models. Eventually, however, consistent predictions of AGB yields and properties should be provided by full stellar model calculations.

\section{Light elements}

The light elements up to boron are easily burned at temperatures of a few $10^{6} \mathrm{~K}$ (with the exception of $\mathrm{H}$ and ${ }^{4} \mathrm{He}$ of course). As a result, they are essentially destroyed by stellar processing. Exceptions, however, are ${ }^{3} \mathrm{He}$ (of importance in relation with Big Bang nucleosynthesis) and ${ }^{7} \mathrm{Li}$ (of interest for Galactic chemical evolution). ${ }^{3} \mathrm{He}$ is discussed in more details in the next paragraphs, while ${ }^{7} \mathrm{Li}$ is developed in [59].

${ }^{3} \mathrm{He}$ : Standard stellar models predict that LIM stars are net producers of ${ }^{3} \mathrm{He}$ (Fig. 1, see also $[88,90]$ ). Two nucleosynthesis paths lead to the production of ${ }^{3} \mathrm{He}$ ([57]): the burning of the initial $\mathrm{D}$ during the pre-main sequence phase through $\mathrm{D}(\mathrm{p}, \gamma)^{3} \mathrm{He}$, and the p-p chain of H-burning during the main sequence through $\mathrm{H}\left(\mathrm{p}, \mathrm{e}^{+} \nu\right) \mathrm{D}(\mathrm{p}, \gamma)^{3} \mathrm{He}$. First dredge-up then mixes the produced ${ }^{3} \mathrm{He}$ in the envelope, which thereafter remains unaltered according to standard models, except in the most massive AGB stars where it is burned by HBB. 
The high ${ }^{3} \mathrm{He}$ abundance observed in the planetary nebula NGC3242 ([70]), of ${ }^{3} R_{3242}=50-100\left[{ }^{3} R \equiv\left({ }^{3} \mathrm{He} / \mathrm{H}\right) \times 10^{5}\right.$, in numbers $]$, supports the standard model predictions ${ }^{2}$. The same conclusion seems true from measurements in evolved stars $([75,46,45])$ (but chemical diffusion in those stars may have increased their surface ${ }^{3} \mathrm{He} /{ }^{4} \mathrm{He}$ ratio). Moreover, the existence of super lithium-rich stars (see discussion in [59]) implies high ${ }^{3} \mathrm{He}$ abundances in their envelope. Taking into account such ${ }^{3} \mathrm{He}$ production by LIM stars, galactic chemical evolution models predict a continuous increase of the interstellar ${ }^{3} \mathrm{He}$ abundance during the course of Galactic evolution.

This, however, is in obvious contradiction with other ${ }^{3} \mathrm{He}$ abundance determinations: (a) ${ }^{3} R_{H I I}=2.42 \pm 1.45$ in $H I I$ regions ${ }^{3}([69,7,9])$, considered as representative of the present-day abundance; (b) ${ }^{3} R_{\odot}=1.48 \pm 0.13$ in the pre-solar nebula, i.e. at the time of solar formation, as inferred from several measurements in meteorites (see Appendix A of [36]); (c) ${ }^{3} R_{I S M}=2.1_{-0.8}^{+0.9}$ in the local interstellar medium, as recently measured from isotopic analysis of helium atoms that have entered the Solar System from the surrounding interstellar cloud ([39]). These low values all suggest that LIM stars produce only mildly, if at all, ${ }^{3} \mathrm{He}^{4}$. Moreover, comparison of ${ }^{3} R_{\odot}$ and ${ }^{3} R_{I S M}$ reveals that ${ }^{3} \mathrm{He}$ has not been much altered during the past $4.6 \mathrm{Gyr}$. Actually, abundance determinations of other elements at the surface of red giants strongly suggest that non-standard mixing occurs during the RGB $([77,82,24,48,25])$ and/or the $\operatorname{AGB}([16,89,1])$ phases in at least a category of LIM stars. These processes are also expected to destroy ${ }^{3} \mathrm{He}$, and galactic chemical evolution models including such destruction are able to account for the above-cited observations (e.g. [66]). But then, the high ${ }^{3} \mathrm{He}$ abundances in planetary nebulae becomes problematic! A solution is to consider that a fraction of LIM stars (10\% is suggested by [66]) are still net ${ }^{3} \mathrm{He}$ producers. Abundance determination in a greater sample of planetary nebulae would help to shed some light on this issue (see review by [71]).

\footnotetext{
${ }^{2}$ Five other planetary nebulae have been analyzed for the presence of ${ }^{3} \mathrm{He}$. Although only upper limits to their ${ }^{3} \mathrm{He}$ abundance are reported, the authors [8] convincingly argue that ${ }^{3} \mathrm{He}$ is also present in at least one or two of them, with ${ }^{3} R_{6543} \lesssim 49 \pm 27$ in NGC6543 and ${ }^{3} R_{7009} \lesssim 69 \pm 27$ in NGC7009.

${ }^{3}$ It is interesting to note that the highest abundance sources are all located in the Perseus arm at $\sim 11 \mathrm{kpc}$ from the galactic center, with an average ${ }^{3} R_{H I I, P e r s}=3.32 \pm$ 1.49 , while the inner galaxy sources have an average ${ }^{3} R_{H I I, \text { in }}=1.45 \pm 0.70$ ([9]).

${ }^{4}$ Starting with a Big Bang Nucleosynthesis value of ${ }^{3} R_{B B N} \simeq 2$ ([72]) and taking into account the ${ }^{3} \mathrm{He}$ production by LIM stars, galactic chemical evolution models predict an overproduction of ${ }^{3} \mathrm{He}$ with respect to the solar value even if only the $\mathrm{D}(\mathrm{p}, \gamma){ }^{3} \mathrm{He}$ production channel is considered in stellar yields ([67]).
} 


\section{CNO elements}

${ }^{12} \mathrm{C}$ : This element is destroyed by H-burning in the stellar interior. Its surface abundance thus slightly decreases during the 1DUP (See Fig. 1), but should remain constant, according to standard evolutionary models, during the ascent of the RGB. Yet, observations of ${ }^{12} \mathrm{C}$ among globular cluster giants reveal a progressive decrease of the $\mathrm{C} / \mathrm{H}$ ratio from the base to the tip of the giant branch by 1.0 dex or more ([82] and references therein). Evidently, some extra-mixing process must occur in those stars during their ascent of the RGB.

${ }^{12} \mathrm{C}$ is also the main product of He-burning in AGB stars through the $3-\alpha$ reactions ( $\sim 20 \%$ in the intershells, in mass fraction). Its mixing in the envelope by the 3DUPs scenario is responsible for the increasing $\mathrm{C} / \mathrm{O}$ ratio observed in the sequence of $\mathrm{M}, \mathrm{MS}, \mathrm{S}, \mathrm{SC}$ and $\mathrm{C}$ stars $(\mathrm{C} / \mathrm{O}>1.0$ in $\mathrm{C}$ stars). As discussed in Sect. 2, AGB models encounter many difficulties in reproducing this scenario consistently and in agreement with observed properties of S and C stars (see [57]). It is also interesting to note that the $\mathrm{C} / \mathrm{O}$ ratio in $\mathrm{C}$ stars does not exceed $\sim 1.6$, with about $50 \%$ of them having $1.0<\mathrm{C} / \mathrm{O} \lesssim 1.1$ (see [54]). It is not clear why this should be so. Apparently, the 3DUP efficiency and/or the total number of pulses experienced during the AGB phase seem to limit the total amount of $\mathrm{C}$ dredged-up to the surface during the whole AGB phase. Stellar mass loss may play a key role in this issue. Observations of the $\mathrm{C}$ abundance in a larger sample of $\mathrm{C}$ stars are welcomed to better constrain the models.

Secondary ${ }^{13} \mathrm{C}$ : Secondary ${ }^{5}{ }^{13} \mathrm{C}$ is produced in stellar interiors by $\mathrm{p}$ capture on the initial ${ }^{12} \mathrm{C}$ through ${ }^{12} \mathrm{C}(\mathrm{p}, \gamma){ }^{13} \mathrm{C}$. First dredge-up then mixes this element in the envelope of red giants. The second dredge-up and 3DUPs do not alter the surface ${ }^{13} \mathrm{C}$ abundance anymore significantly. However, HBB in the most massive AGB stars further transforms ${ }^{12} \mathrm{C}$ into ${ }^{13} \mathrm{C}$, eventually decreasing the surface ${ }^{12} \mathrm{C} /{ }^{13} \mathrm{C}$ ratio down to the $\mathrm{CN}$ equilibrium value of $3-4$.

The ${ }^{12} \mathrm{C} /{ }^{13} \mathrm{C}$ ratio is a good tracer of the evolution of both ${ }^{12} \mathrm{C}$ and ${ }^{13} \mathrm{C}$. Its predicted value after the 1DUP lies between 20 and 30 (Fig. 1, solar value $=90$ ). This is, in general, in good agreement with the observed ratio in intermediate-mass red giants ([31] and references therein). However, many other observed ${ }^{12} \mathrm{C} /{ }^{13} \mathrm{C}$ ratios fall below the standard model predictions. These include, for example, those at the surface of low-mass Pop. I field giants (e.g. [31] and references therein), of red giants in Galactic open clusters (e.g. $[19,37,38])$ or in globular clusters (e.g. [83, 20, 18]), and of metal-poor

\footnotetext{
${ }^{5}$ An element is said to be 'primary' if it can be synthesized in a star of the first generation (i.e. with zero metallicity), and 'secondary' if it needs the presence of some elements heavier than $\mathrm{He}$ in the initial stellar chemical composition.
} 
field stars (e.g. [84]). They require some non-standard mechanism (most probably mixing) to operate in low-mass stars (see, e.g. [77, 37, 38]). Two observational works should be mentioned. First, the analysis of the ${ }^{12} \mathrm{C} /{ }^{13} \mathrm{C}$ ratio in 20 Galactic open clusters as a function of their turn-off mass $M_{\text {toff }}$ reveals that this ratio is $\sim 25$ for $\mathrm{M}_{\text {toff }} \gtrsim 2.2 \mathrm{M}_{\odot}$, as expected, but decreases below $2.2 \mathrm{M}_{\odot}$, reaching $\sim 10$ at $\mathrm{M}_{\text {toff }} \simeq 1.2 \mathrm{M}_{\odot}{ }^{6}([37])$. Second, the analysis of the distribution of ${ }^{12} \mathrm{C} /{ }^{13} \mathrm{C}$ along the giant branch of the open cluster $\mathrm{M} 67$ (which has $\mathrm{M}_{\text {toff }} \simeq 1.2 \mathrm{M}_{\odot}$ ) reveals that this ratio is compatible with standard model predictions for stars located at the bottom of the $\mathrm{RGB}$, while those at the tip exhibit anomalously low ratios ([38]). As in the case of ${ }^{12} \mathrm{C}$ in globular clusters, extra-mixing during the ascent of the RGB would explain the ${ }^{12} \mathrm{C} /{ }^{13} \mathrm{C}$ pattern along the RGB of M67 ([24]). More observations are, however, needed to better characterize the stars which show evidences of such process. This has consequences on abundance predictions of other elements as well, such as ${ }^{3} \mathrm{He}$ which is expected to be partially (totally?) destroyed by the extra-mixing ([48, 25]).

During the AGB phase, the ${ }^{12} \mathrm{C} /{ }^{13} \mathrm{C}$ ratio is expected to increase as a result of the surface ${ }^{12} \mathrm{C}$ enhancement brought by the 3DUPs, by a factor up to 4 in $\mathrm{C}$ stars having $\mathrm{C} / \mathrm{O} \simeq 1.2$. The ratios observed in AGB stars agree well with these expectations, ranging from $\sim 5$ to $\sim 50$ in MS, S and SC stars $([79,30,29,80])$, and from $\sim 30$ to $\sim 70$ in $\mathrm{C}$ stars $([54,65,28])$ (see also $[52,51,5,6])$.

Let me finally mention the special class of $\mathrm{C}$ stars called $\mathrm{J}$ stars (e.g. $[54,33])$, which exhibit very low ${ }^{12} \mathrm{C} /{ }^{13} \mathrm{C}$ ratios, down to $3-4$ ([43]), and which could be identified with AGB stars undergoing HBB ([10]). The high ${ }^{16} \mathrm{O} /{ }^{18} \mathrm{O}$ ratios observed in those stars support this idea (though some normal $\mathrm{C}$ stars have also similar high oxygen isotopic ratios, see sections on ${ }^{16} \mathrm{O},{ }^{17} \mathrm{O}$ and ${ }^{18} \mathrm{O}$ ). However, their $\mathrm{N}$ abundances and $\mathrm{C} / \mathrm{O}$ ratios are not in agreement with HBB expectations. We refer to [43] for further discussion on these objects.

Primary ${ }^{13} \mathrm{C}$ : Primary ${ }^{13} \mathrm{C}$ can be produced in AGB stars in two different ways. The first way is evidenced by the observations of both fluorine and s-process elements in $\mathrm{S}$ to $\mathrm{C}$ stars $([50])$, which are synthesized in the Heburning shell in the presence of neutrons. These neutrons are believed to be produced by ${ }^{13} \mathrm{C}(\alpha, \mathrm{n}){ }^{16} \mathrm{O}$. A primary source of ${ }^{13} \mathrm{C}$ is necessary because the amount of ${ }^{13} \mathrm{C}$ left over by the H-burning shell in Pop. I stars is too low by a factor of at least 30 in order to match observations of $\mathrm{F}$ and s-process elements $([61])$. The production of such primary ${ }^{13} \mathrm{C}$ (and ${ }^{14} \mathrm{~N}$ ) has long been a challenge for AGB modelers $([74])$, but looks more promising today

\footnotetext{
${ }^{6}$ The very low ${ }^{12} \mathrm{C} /{ }^{13} \mathrm{C}$ ratios of $3-5$ in globular clusters, which have $\mathrm{M}_{\mathrm{toff}}<1.0$, enter well into this picture (see Fig. 6 of [24]).
} 
([47]). This primary ${ }^{13} \mathrm{C}$ cannot, however, account for any stellar surface, and thus interstellar, ${ }^{13} \mathrm{C}$ enrichment since it is burned in the He-burning layers before the operation of 3DUP.

Another source of primary ${ }^{13} \mathrm{C}$ (and $\left.{ }^{14} \mathrm{~N}\right)$ is provided in the envelope of those AGB stars undergoing HBB ([68]), by H-burning of the primary ${ }^{12} \mathrm{C}$ dredged-up during 3DUP scenarios. Unfortunately, the shortcomings affecting AGB models with respect to 3DUP and $\mathrm{HBB}$ do not allow presently to provide consistent primary ${ }^{13} \mathrm{C}\left(\right.$ and ${ }^{14} \mathrm{~N}$ ) yields from AGB stars.

Secondary ${ }^{14} \mathrm{~N}$ : Being the main product of the CNO cycles of H-burning, ${ }^{14} \mathrm{~N}$ is largely enhanced after the 1DUP. This is well confirmed by observations, which moreover confirm the expected anti-correlation between $\mathrm{N}$ and both $\mathrm{C}$ and $\mathrm{O}$ (e.g. [81]). The 3DUP, on the other hand, does not affect ${ }^{14} \mathrm{~N}$ which is not produced during helium burning. HBB, however, can further enhance the ${ }^{14} \mathrm{~N}$ surface abundance in AGB stars.

Primary ${ }^{14} \mathrm{~N}$ : Large amounts of primary ${ }^{14} \mathrm{~N}$ can be produced by HBB in the envelope of AGB stars after primary ${ }^{12} \mathrm{C}$ has been dredged-up from the He-burning layers ([68]). Like for primary ${ }^{13} \mathrm{C}$, however, no consistent yield of primary ${ }^{14} \mathrm{~N}$ is provided yet by current AGB models.

${ }^{15} \mathrm{~N}$ : The NO cycles essentially destroy ${ }^{15} \mathrm{~N}$. Its abundance at the surface of red giants is determined by the degree of dilution it undergoes when the envelope deepens in the stellar interior. As a result, the ${ }^{14} \mathrm{~N} /{ }^{15} \mathrm{~N}$ ratio increases during the 1DUP from 270 to $400-1050$ in Pop. I stars, depending of the stellar mass (Fig. 1; solar value $=270$ ).

Primary ${ }^{15} \mathrm{~N}$ : This nuclide is produced in the He-burning shell of AGB stars (see [60]). Its synthesis results from ${ }^{14} \mathrm{~N}(\alpha, \gamma){ }^{18} \mathrm{~F}\left(\beta^{+}\right){ }^{18} \mathrm{O}(\mathrm{p}, \alpha){ }^{15} \mathrm{~N}$, where the required protons are provided by ${ }^{14} \mathrm{~N}(\mathrm{n}, \mathrm{p}){ }^{14} \mathrm{C}$ and the neutrons by ${ }^{13} \mathrm{C}(\alpha, \mathrm{n}){ }^{16} \mathrm{O}$. As both ${ }^{13} \mathrm{C}$ and ${ }^{14} \mathrm{~N}$ required for that synthesis are primary (see section on primary ${ }^{13} \mathrm{C}$ ), the resulting ${ }^{15} \mathrm{~N}$ is also primary. Nitrogen eventually burns into fluorine through ${ }^{15} \mathrm{~N}(\alpha, \mathrm{p}){ }^{19} \mathrm{~F}$. According to evolutionary AGB models ([60]), however, the transformation of ${ }^{15} \mathrm{~N}$ into ${ }^{19} \mathrm{~F}$ is only partial during the first pulses, and becomes more complete as the temperature in the He-burning shell increases. Primary ${ }^{15} \mathrm{~N}$ could then be brought to the surface if the 3DUP operates early enough along the AGB phase. Again, due to the difficulties of current AGB models to predict both primary ${ }^{13} \mathrm{C}$ and the 3DUP scenario (see Sect. 2), no consistent yield for primary ${ }^{15} \mathrm{~N}$ (if it is indeed dredged-up to the surface) is available yet.

${ }^{16} \mathrm{O}:{ }^{16} \mathrm{O}$ is partly destroyed by the NO cycle, but its abundance at the surface of red giants is not much altered by the 1DUP (see Fig. 1). 
The effect of the 3DUPs on that element is less clear. Determinations of the ${ }^{16} \mathrm{O} /{ }^{17} \mathrm{O}$ and ${ }^{16} \mathrm{O} /{ }^{18} \mathrm{O}$ ratios at the surface of $\mathrm{MS}, \mathrm{S}$ and $\mathrm{C}$ stars $([42,43])$ reveal values $(600-4000)$ much higher than those in normal red giants (100-700). The simplest explanation for these high ratios invokes an enhancement of ${ }^{16} \mathrm{O}$ by 3DUP. This scenario seems to be supported by the relative constancy of the ${ }^{17} \mathrm{O} /{ }^{18} \mathrm{O}$ ratio in those stars (see Table 2 of [27]). From the theoretical point of vue, such a scenario is possible if enough ${ }^{16} \mathrm{O}$ is created in the He-burning shell through ${ }^{12} \mathrm{C}(\alpha, \gamma){ }^{16} \mathrm{O}$. However, the predicted oxygen composition in the He-burning shell of AGB models is $\mathrm{X}\left({ }^{16} \mathrm{O}\right) \simeq 1 \%$, i.e. comparable to that in the envelope. As a result no surface ${ }^{16} \mathrm{O}$ enhancement is predicted to occur during the 3DUP. And in fact, the ${ }^{16} \mathrm{O}$ abundances measured in $\mathrm{C}$ stars are essentially similar to those in $\mathrm{M}$ and MS giants ([54]). The oxygen isotopic ratios remain thus an intriguing puzzle for stellar modelers.

${ }^{17} \mathrm{O}:{ }^{17} \mathrm{O}$ is produced in the NO cycle of $\mathrm{H}$-burning, in amounts very dependent on the temperature at which hydrogen burns. Figure 2 of [4] indicates that the maximum ${ }^{17} \mathrm{O}$ production occurs at temperatures around $17 \times 10^{6} \mathrm{~K}$. Because temperatures in stellar interiors increase with the stellar mass, a large scatter is expected to characterize the ${ }^{17} \mathrm{O}$ abundances at the surface of red giants as well. Figure 1 indicates that the maximum enhancement of ${ }^{17} \mathrm{O}$ after the 1DUP is found for stars with masses between 2.2 and $2.5 \mathrm{M}_{\odot}$. Concomitantly, the predicted ${ }^{16} \mathrm{O} /{ }^{17} \mathrm{O}$ ratio after the $1 D U P$ varies from 100 to 600 depending on the stellar mass. The second dredge-up does not alter the ${ }^{17} \mathrm{O}$ abundance much further.

A large scatter in the ${ }^{16} \mathrm{O} /{ }^{17} \mathrm{O}$ ratio is indeed observed in red giants $([40,44,21])$, which can be ascribed to differences in stellar masses in agreement with model predictions. It is interesting to note that, in contrast to ${ }^{16} \mathrm{O} /{ }^{17} \mathrm{O}$, the ${ }^{12} \mathrm{C} /{ }^{13} \mathrm{C}$ ratios in those red giants fall below the expected values. The extra-mixing required to explain the low carbon isotopic ratios in those stars should thus operate only down to layers between the ${ }^{13} \mathrm{C}$-rich and ${ }^{17} \mathrm{O}$-rich zones.

As for the ${ }^{17} \mathrm{O}$ abundance in AGB stars, standard models predict no alteration during a $3 \mathrm{DUP}$, while $\mathrm{HBB}$ produces ${ }^{17} \mathrm{O}([16])$. The high ${ }^{16} \mathrm{O} /{ }^{17} \mathrm{O}$ ratios observed in $\mathrm{MS}, \mathrm{S}$ and $\mathrm{C}$ stars, and which could not be explained by a ${ }^{16} \mathrm{O}$ enhancement (see Sect. on ${ }^{16} \mathrm{O}$ ), remain thus without any explanation ${ }^{7}$ ! I refer to [27] for further discussion. More observations of the isotopic ratios in AGB stars are welcomed.

\footnotetext{
${ }^{7} \mathrm{The}{ }^{16} \mathrm{O} /{ }^{17} \mathrm{O}$ ratios observed in a few SC stars (between 75 and $1200,[30,29]$ ) are lower than those observed in both $\mathrm{S}$ and $\mathrm{C}$ stars. If these values are confirmed, they raise a conceptual difficulty since the evolutionary state of SC stars is believed to lie between those of $\mathrm{S}$ and $\mathrm{C}$ stars.
} 
${ }^{18} \mathrm{O}:{ }^{18} \mathrm{O}$ is destroyed during the NO cycles. As a result, a slight increase in the surface ${ }^{16} \mathrm{O} /{ }^{18} \mathrm{O}$ ratio is expected to occur after the 1DUP (Fig. 1). This increase, however, is smaller than the observational error bars on that ratio (see, e.g., Fig. 7a of [31]), and no conclusion can be drawn from the comparison of observations with predictions.

In AGB stars, ${ }^{18} \mathrm{O}$ is not expected to be altered by 3DUP according to standard models. The ${ }^{16} \mathrm{O} /{ }^{18} \mathrm{O}$ ratio should thus remain unchanged ${ }^{8}$. The high ${ }^{16} \mathrm{O} /{ }^{18} \mathrm{O}$ ratios observed in MS, $\mathrm{S}$ and $\mathrm{C}$ stars raises difficulties similar to those from ${ }^{16} \mathrm{O} /{ }^{17} \mathrm{O}$ (see Sect. on ${ }^{16} \mathrm{O} /{ }^{17} \mathrm{O}$ ).

${ }^{18} \mathrm{O}$ can also be destroyed by $\mathrm{HBB}$, resulting in an increase of the ${ }^{16} \mathrm{O} /{ }^{18} \mathrm{O}$ ratio by several orders of magnitude ([16]). Such high values should characterize the ${ }^{13} \mathrm{C}$-rich $\mathrm{J}$ stars (see Sect. on ${ }^{13} \mathrm{C}$ ).

\section{Fluorine}

The observation of large ${ }^{19} \mathrm{~F}$ over-abundances in $\mathrm{S}$ and $\mathrm{C}$ stars relative to those in normal giants ([50]) strongly suggests the production of that element in AGB stars. The existence of a correlation between ${ }^{19} \mathrm{~F}$ and $\mathrm{s}$ process elements nucleosynthesis is moreover established on both observational ([50]) and theoretical ([61]) grounds. Stellar evolutionary calculations indeed confirm the production of ${ }^{19} \mathrm{~F}$ in AGB models $([34,60])$, but require an additional source of primary ${ }^{13} \mathrm{C}$. Like the s-process elements thus, fluorine predictions suffer from the inability of current AGB models to produce consistently primary ${ }^{13} \mathrm{C}$ and the $3 \mathrm{DUP}$ scenario. We refer to the abovecited references for further discussion on the fluorine issue in red giants (see also [62] in this volume).

Let us also remark that secondary ${ }^{19} \mathrm{~F}$ could be produced during $\mathrm{H}$ burning by ${ }^{18} \mathrm{O}(\mathrm{p}, \gamma){ }^{19} \mathrm{~F}([4])$. Stellar models however, predict only very little enhancement of that element after the 1DUP in LIM stars (Fig. 1; see also discussion in [60]).

\section{Conclusions and final remarks}

This short review has focused on the discrepancies between the predicted and observed abundances at the surface of both RGB and AGB stars. It is

\footnotetext{
${ }^{8}$ Actually, ${ }^{18} \mathrm{O}$ is produced by ${ }^{14} \mathrm{~N}(\alpha, \gamma){ }^{18} \mathrm{O}$ in the He-burning shell, ${ }^{14} \mathrm{~N}$ being provided by the ashes of the H-burning shell, and destroyed by ${ }^{18} \mathrm{O}(\alpha, \gamma){ }^{22} \mathrm{Ne}$. Evolutionary models show that the destruction is only partial during the first pulses where the temperatures are lower than about $2.2 \times 10^{8} \mathrm{~K}$. As a result, ${ }^{18} \mathrm{O}$ could be mixed to the surface by a 3DUP if the latter occurs during the very first pulses. No current AGB model confirms this scenario, though. However, one star, the barium star HD101013, presents a ${ }^{16} \mathrm{O} /{ }^{18} \mathrm{O}$ ratio as low as $60_{-30}^{+100}([41])$. If confirmed, it could mean that a (very small) fraction of AGB stars do indeed dredge-up ${ }^{18} \mathrm{O}$ from the He-burning layers.
} 
shown in particular that, while the predicted trends from 'classical' stellar models are well confirmed by the observations, quantitative comparisons put some limitations on the predictive capabilities of such models. Most of the discrepancies suggest that mixing occurs in the stellar interior deeper than that predicted by classical models. In addition, chemical abundance predictions from presently available AGB models further suffer from the uncertainties affecting the third dredge-up phenomenon, the source of neutrons and the hot bottom burning process.

Finally I must mention a new class of objects which have been analyzed during the last decade, and which provide valuable information on the chemical abundances ejected by stars. These are inclusions in primitive meteorites which show anomalous isotopic compositions (i.e. different, often by large amounts, from the relatively homogeneous abundances reported in material of Solar System origin). The currently accepted explanation for these anomalies considers that these grains are of stellar origin, and have been injected in the Solar System during its formation. The fact that SiC grains have also been detected in circumstellar envelopes of carbon stars through their $11.4 \mu \mathrm{m}$ emission feature (e.g. [53] and references therein) strongly supports that scenario. I refer to $[2,91,63]$ for some general reviews on the interstellar grains from primitive meteorites and on their isotopic composition. In particular, it is interesting to note that the analysis of the oxygen isotopic ratios in those grains supposed to have originated from AGB stars leads - again - to the conclusion that extra-mixing must have operated in those stars $([15,64])$. Such studies nicely complement the direct isotopic abundance determinations from the light spectrum of red giants.

Acknowledgment: I thank Drs. Alain Jorissen and Georges Meynet for their careful reading and commenting on the manuscript.

\section{References}

1. Abia C., Isern J.: 1997, MNRAS 289, L11

2. Anders E., Zinner E.: 1993, Meteoritics 28, 490

3. Arnould M.: 1991, in 'Evolution of Stars: The Photospheric Abundance Connection' (IAU Symp. 145), eds. Michaud G, and Tutukov A., Kluwer, p 287

4. ${ }^{9}$ Arnould M., Mowlavi N., Champagne A.: 1996, in 32nd Liège Int. Astroph. Coll., eds. Noels A., Fraipont-Caro D., Gabriel M., Grevesse N., Demarque P., pp 17-29

5. Bakker E.J., Van Dishoeck E.F., Waters L.B.F.M., Schoenmaker T.: 1997, A\&A 323,469

6. Bachiller R., Forveille T., Huggins P.J., Cox P.: 1997, A\&A 324, 1123

7. Bania T.M., Rood R.T., Wilson T.L.: 1987, ApJ 323, 30

8. Balser D.S., Bania T.M., Rood R.T., Wilson T.L.: 1997, ApJ 483, 320

\footnotetext{
${ }^{9}$ postscript files available by anonymous ftp on 'obsftp.unige.ch' in 'pub/mowlavi', or in 'http://obswww.unige.ch/ mowlavi/publications/publications.html
} 
9. Balser D.S., Bania T.M., Brockway C.J., Rood R.T., Wilson T.L.: 1994, ApJ 430, 667

10. Becker S.A., Iben I.Jr.: 1980, ApJ 237, 111

11. Bernasconi P.A.: 1996, A\&AS 120, 57

12. Blackmon J.C., Champagne A.E., Hofstee M.A., Smith M.S., Downing R.G., Lamaze G.P.: 1995, Phys. Rev. Lett. 74, 2642

13. Blöcker T.: 1995, A\&A 297, 727

14. Boothroyd A.I., Sackmann I.-J.: 1988, ApJ 328, 653

15. Boothroyd A.I., Sackmann I.-J., Wasserburg G.J.: 1994, ApJ 430, L77

16. Boothroyd A.I., Sackmann I.-J., Wasserburg G.J.: 1995, ApJ 442, L21

17. Bressan A., Fagotto F., Bertelli G., Chiosi C.: 1993, A\&AS 100, 647

18. Briley M.M., Smith V.V., Lambert D.L.: 1994, ApJ 424, L119

19. Brown J.A.: 1987, ApJ 317, 701

20. Brown J.A., Wallerstein G.: 1989, AJ 98, 1643

21. Campbell B., Lambert D.L., Maillard J.-P.: 1990, PASP 102, 79

22. Canuto V.M., Goldman I., Mazzitelli I.: 1996, ApJ 473, 550

23. Castellani V., Chieffi A., Straniero O.: 1990, ApJS 74, 463

24. Charbonnel C.: 1994, A\&A 282, 811

25. Charbonnel C.: 1995, ApJ 453, L41

26. Coc A., Mochkovitch R., Oberto Y., Thibaud J.P., Vangioni-Flam E.: 1995, A\&A 299,479

27. Dearborn D.S.P: 1992, Phys. Reports 210, 367

28. de Laverny P., Gustafsson B.: 1998, A\&A, in press

29. Dominy J.F., Wallerstein G.: 1987, ApJ 317, 810

30. Dominy J.F., Wallerstein G., Suntzeff N.B.: 1986, ApJ 300, 325

31. El Eid M.F.: 1994, A\&A 285, 915

32. El Eid M.F., Champagne A.E.: 1995, ApJ 451, 298

33. Lloyd E.T..: 1995, Astr.Sp.Sci. 230, 169

34. Forestini M., Goriely S., Jorissen A., Arnould M.: 1992, A\&A 261, 157

35. Forestini M., Charbonnel C.: 1997, A\&AS 123, 241

36. Galli D., Palla F., Ferrini F., Penco U.: 1995, ApJ 443, 536

37. Gilroy K.K.: 1989, ApJ 347, 835

38. Gilroy K.K., Brown J.A.: 1991, ApJ 371, 578

39. Gloeckler G., Geiss J.: 1996, Nature 381, 210

40. Harris M.J., Lambert D.L.: 1984, ApJ 285, 674

41. Harris M.J., Lambert D.L., Smith V.V.: 1985, ApJ 292, 620

42. Harris M.J., Lambert D.L., Smith V.V.: 1985, ApJ 299, 375

43. Harris M.J., Lambert D.L., Hinkle K.H., Gustafsson B., Eriksson K.: 1987, ApJ 316,294

44. Harris M.J., Lambert D.L., Smith V.V.: 1988, ApJ 325, 768

45. Hartoog M.R.: 1979, ApJ 231, 161

46. Hartoog M.R., Cowley A.P.: 1979, ApJ 228, 229

47. Herwig F., Blöcker T., Schönberner D., El Eid M.: 1997, A\&A 324, L81

48. Hogan 1995: ApJ 441, L17

49. Iben I.Jr.: 1981, ApJ 246, 278

50. Jorissen A., Smith V.V., Lambert D.L.: 1992, A\&A 261, 164

51. Kahane C., Cernicharo J., Gomez-Gonzales J., Guelin M.: 1992, A\&A 256, 235

52. Kahane C., Gomez-Gonzales J., Cernicharo J., Guelin M.: 1988, A\&A 190, 167

53. Kozasa T., Dorschner J., Henning T., Stognienko R.: 1996, A\&A 307, 551

54. Lambert D.L., Gustafsson B., Eriksson K., Hinkle K.H.: 1986, ApJS 62, 373

55. Marigo P., Bressan A., Chiosi C.: 1996, A\&A 313, 545

56. ${ }^{9}$ Mowlavi N.: 1995, Ph.D. thesis, unpublished

57. ${ }^{9}$ Mowlavi N.: 1998, in 'Tours Symposium on Nuclear Physics III', ed. H. Utsunomiya, AIP, in press

58. ${ }^{9}$ Mowlavi N.: 1998, in 'Abundance profiles: Diagnostic Tools for Galaxy History', 
eds. Friedli D., Edmunds M.G., Robert C., Drissen L., ASP Conf. Ser., in press

59. ${ }^{9}$ Mowlavi N.: 1998, in 'Pacific RIM Conference on Stellar Astrophysics', eds. Chan K.L., Cheng K.S., Singh H.P., PASP Conf. Proc., in press

60. ${ }^{9}$ Mowlavi N., Jorissen A., Arnould M: 1996, A\&A 311, 803

61. ${ }^{9}$ Mowlavi N., Jorissen A., Arnould M: 1998, A\&A, submitted

62. ${ }^{9}$ Mowlavi N., Meynet G., this volume

63. Nittler L.R.: 1997, in 'Astrophysical Implications of the Laboratory Study of Presolar Materials', eds. Bernatowicz, Zinner E., AIP, New York, p 59

64. Nittler L.R., Alexander C.M., Gao X, Walker R.M., Zinner E.: 1997, ApJ 483, 475

65. Ohnaka K., Tsuji T.: 1996, A\&A 310, 933

66. Olive K.A., Schramm D.N., Scully S.T., Truran J.W.: 1997, ApJ 479, 752

67. Prantzos N.: 1996, A\&A 310, 106

68. Renzini A., Voli M.: 1981, A\&A 94, 175

69. Rood R., Bania T.M., Wilson T.L.: 1984, ApJ 280, 629

70. Rood R., Bania T.M., Wilson T.L.: 1992, Nature 355,618

71. Rood R., Bania T.M., Balser D.S., Wilson T.L.: 1997, to appear in 'International Light Elements Workshop', Bern

72. Reeves H.: 1974, ARAA 12, 437

73. Rogers F.J., Swenson F.J., Iglesias C.A.: 1996, ApJ 456, 902

74. Sackmann I.-J., Boothroyd A.I., in 'Evolution os Stars: The Photospheric Abundance Connection' (IAU Symp. 145), eds. Michaud G, and Tutukov A., Kluwer, Dordrecht, p 275

75. Sargent W.L.W., Jugaku J.: 1961, ApJ 134, 777

76. Schaller G., Schaerer D., Meynet G., Maeder A.: 1992, A\&AS 96, 269

77. Sweigart A.V., Mengel J.G.: 1979, ApJ 229, 624

78. Smith V.V.: 1989, in 'Cosmic Abundances of matter', ed. Waddington C.J., AIP Conf. Proc. 183, p 200

79. Smith V.V., Lambert D.L.: 1985, ApJ 294, 326

80. Smith V.V., Lambert D.L.: 1985, ApJS 72, 387

81. Smith G.H., Shetrone M.D., Bell R.A., Churchill C.W., Briley M.M.: 1996, AJ 112, 1511

82. Smith G.H., Tout C.A.: 1992, MNRAS 256, 449

83. Smith V.V.,Suntzeff N.B.: 1989, AJ 97, 1699

84. Sneden C., Pilachowski C.A., Vandenberg D.A.: 1986, ApJ 311, 826

85. Straniero O., Chieffi A., Limongi M., Busso M., Gallino R., Arlandini C.: 1997, ApJ 478,332

86. VandenBerg D.A.: 1992, ApJ 391, 685

87. van den Hoek L.B., Groenewegen M.A.T.: 1997, A\&AS 123, 305

88. Vassiliadis E., Wood P.R.: 1993, ApJ 413, 641

89. Wasserburg G.J., Boothroyd A.I., Sackmann I.-J.: 1995, ApJ 447, L37

90. Weiss A., Wagenhuber J., Denissenkov P.A.: 1996, A\&A 313, 581

91. Zinner E.: 1994, Meteoritics 29, 555 